\title{
EDITORIAL
}

\section{Gestational diabetes mellitus: what is the optimal treatment modality?}

Journal of Perinatology (2007) 27, 257-258. doi:10.1038/sj.jp.7211737

Gestational diabetes mellitus (GDM) is characterized by carbohydrate intolerance that is first recognized or begins during pregnancy. Its prevalence varies widely depending on the study population; commonly, it has been reported to range between 2 and $5 \%$ in the United States. ${ }^{1}$ It has been recognized that pregnancies complicated by GDM is associated with higher incidences of spontaneous preterm delivery, ${ }^{2}$ pre-eclampsia and cesarean delivery compared to women without a diagnosis of $\mathrm{GDM}^{3}$ Neonatal complications associated with GDM also include macrosomia with its associated complications such as shoulder dystocia and birth trauma, hyperbilirubinemia and hypoglycemia. ${ }^{1}$ Although the benefit of glycemic control in women diagnosed with GDM seems intuitive, the causal association between hyperglycemia and perinatal complications was only recently validated by a randomized controlled study. Crowther $e t$ al. ${ }^{4}$ demonstrated lower rates of pre-eclampsia, macrosomia, admissions to the neonatal intensive care nursery and composite perinatal complications that included death, shoulder dystocia, bone fracture and nerve palsy in the group of women diagnosed with GDM who received dietary counseling, glucose monitoring and treatment if indicated in the intervention group, compared to the control group with similar background characteristics who received routine care.

The management goals of gestational diabetes generally include fetal surveillance and prevention of perinatal complications.

Glycemic control usually begins with diet and exercise and medical therapy if necessary. Although insulin has been the gold standard in the treatment of GDM, in recent years oral hypoglycemic agents (e.g., glyburide) have been considered and utilized as well. ${ }^{1}$ When glyburide was compared with insulin for the treatment of GDM in one randomized controlled trial, the authors reported similar glycemic control and no differences in perinatal outcomes they examined, including cesarean delivery, preeclampsia, macrosomia and neonatal hypoglycemia. ${ }^{5}$ However, with only 200 subjects in each arm, this study did not have adequate statistical power to examine some of these rare outcomes. For example, to find a statistical difference with a frequency of $9 \%$ hypoglycemia in the glyburide group versus the $6 \%$ hypoglycemia in the insulin group, over 1,200 patients in each arm would be necessary despite a 50\% increase of hypoglycemia in the glyburide group.

The American College of Obstetricians and Gynecologists (ACOG) recommendations call for further studies to confirm the efficacy of oral agent 'before the use of newer oral hypoglycemia agents can be supported for use in pregnancy. ${ }^{1}$ However, the utilization of glyburide as an alternative treatment option to insulin in women with GDM has been adopted by many clinicians. A recent poll of obstetricians and gynecologists in 2003 found that $13 \%$ of the fellows prescribe glyburide as the initial therapy for GDM beyond diet. ${ }^{6}$ At the population level, a recent abstract reported that $16 \%$ of women with the need for medical treatment in pregnancy received oral agents. ${ }^{7}$

In this issue of Journal of Perinatology, Ramos et al. ${ }^{8}$ compared the effectiveness of glyburide and insulin for the treatment of GDM in women who had oral glucose challenge test (OGCT) $\geqslant 200 \mathrm{mg} / \mathrm{dl}$ and fasting hyperglycemia. They found that glyburide achieved similar birth weights and delivery outcomes but was associated with an increased risk of macrosomia as well as a positive trend toward neonatal hypoglycemia. Using the same overall cohort comparing glyburide to insulin in women diagnosed with GDM, Jacobson et al. ${ }^{9}$ also found that women who received glyburide were more likely to experience pre-eclampsia, neonatal hyperbilirubinemia requiring phototherapy and longer length of stay in the neonatal intensive care unit compared to women who were treated with insulin. These findings suggest that perhaps glyburide may not be equally efficacious as insulin for the management of GDM requiring medical therapy. Echoing what ACOG stated in 2001, further adequately powered studies are needed to evaluate the efficacy of glyburide as a treatment for pregnancies complicated by GDM. Until that time, we believe that insulin should be considered the first-line medical treatment of diabetes in pregnancy.

YW Cheng and $\mathrm{AB}$ Caughey Department of Obstetrics, Gynecology and Reproductive Sciences, University of California, San Francisco, CA, USA E-mail: abcmd@berkeley.edu

\section{References}

1 America College of Obstetricians and Gynecologists (ACOG) practice bulletin. Clinical management guidelines for obstetrician-gynecologists. Gestational Diabetes. No. 30, September 2001, Washington DC.

2 Hedderson MM, Ferrara A, Sacks DA. Pregnancy hyperglycemia: association with increased risk of spontaneous preterm birth. Am J Obstet Gynecol 2003; 102: $850-856$. 
3 Naylor CD, Sermer M, Chen E, Sykora K. Cesarean delivery in relation to birth weight and gestational glucose tolerance: pathophysiology or practice style? Toronto Trihospital Gestational Diabetes Investigators. JAMA 1996; 275: 1165-1170.

4 Crowther CA, Hiller JE, Moss JR, McPhee AJ, Jeffries WS, Robinson JS, for the Australian Carbohydrate Intolerance Study in Pregnant Women (ACHOIS) Trial Group. Effect of treatment of gestational diabetes mellitus on pregnancy outcomes. N Engl J Med 2005; 352: 2477-2486.

5 Langer 0, Conway DL, Derkus MD, Xenakis EMJ, Gonzales 0. A comparison of glyburide and insulin in women with gestational diabetes mellitus. $N$ Engl J Med 2000; 343: 1134-1138.

6 Gabbe SG, Gregory RP, Power ML, Williams SB, Schulkin J. Management of diabetes mellitus by obstetrician-gynecologists. Obstet Gynecol 2004; 103: 1229-1234.
7 Cheng YW, Chung JH, Block-Kurbisch I, Inturrisi M, Caughey AB. Treatment of gestational diabetes mellitus: oral hypoglycemic agents compared to subcutaneous insulin therapy. Am J Obstet Gynecol 2007; 195: S36.

8 Ramos GA, Jacobson GF, Kirby RS, Ching JY, Field DR. Comparison of glyburide and insulin for the management of gestational diabetics with markedly elevated oral glucose challenge test and fasting hyperglycemia. J Perinatol 2007 (in press)

9 Jacobson GF, Ramos GA, Ching JY, Kirby RS, Ferrara A, Field DR. Comparison of glyburide and insulin for the management of gestational diabetes in a large managed care organization. Am J Obstet Gynecol 2005; 193: $118-124$. 